\title{
DEVELOPMENT OF HIGH CURRENT SURFACE IONIZATION SOURCES FOR HEAVY ION FUSION*
}

\author{
J. W. Kwan, F.M. Bienionsek, E. Chacon-Golcher, D. Baca \\ LBNL, Berkeley, CA 94720, USA
}

\begin{abstract}
Due to their intrinsic low ion temperature, surface ionization sources with a moderate to high current density $\left(\mathrm{J} \sim 10-100 \mathrm{~mA} / \mathrm{cm}^{2}\right)$ may meet the beam brightness requirement for Heavy Ion Fusion (HIF) injectors, for both, the large source and multiple-beamlet designs. However, alkali metals released by these sources can contaminate and degrade high voltage insulators, thus making neutral atom loss an important issue. We present experimental results of average emitted J's and lifetime performance of $\mathrm{K}^{+}$and $\mathrm{Cs}^{+}$surface ionization sources and $\mathrm{K}$-aluminosilicate sources operating in pulsed modes (2$10 \mu \mathrm{s})$. Fabrication of large $(\sim 10 \mathrm{~cm}$ diameter $)$ aluminosilicate sources is challenging given the surface quality required. Current work in this aspect is presented.
\end{abstract}

\section{INTRODUCTION}

The HIF concept intends to use heavy particle beams to drive fusion reactions in a Deuterium-Tritium pellet. Depending on the injector design, different source requirements are involved. On one hand, large sources (multi $-\mathrm{cm}$ diameter) could be used requiring J's of a few $\mathrm{mA} / \mathrm{cm}^{2}$. Other designs involve arrays of small aperture (mm-size) sources with J's of up to $100 \mathrm{~mA} / \mathrm{cm}^{2}$ [1]. For these reasons, research is conducted at LBNL on different types of ion sources that could meet the design requirements of a HIF driver. Regardless of the source used, some general requirements must be met, such as very low beam emittance, low neutral gas emission, and beam spatial and temporal uniformity during the pulse duration. In this paper we discuss general issues related to the making of surface ionization sources that are deemed as good candidates for future HIF experiments, and possibly a future HIF driver. Section 2 discusses the ion sources preparation processes involved. Section 3 presents results obtained in our experiments at LBNL and, section 4 presents the conclusions and future research in this topic.

\section{SOURCE PREPARATION \& OPERATION}

\subsection{Doped Source}

The small sources used at the high current density experiments are manufactured at LBNL by pressing $6 \mu \mathrm{m}$ grain size tungsten powder in a $1 / 4$ " press under a $4 \mathrm{klb}$

\footnotetext{
*Work supported by the Office of Fusion Energy, US DOE under contract No. DE-AC03-76SF00098.
}

load. The buttons are then fired for sintering at $1750{ }^{\circ} \mathrm{C}$ for two hours. These substrates are then doped with a potassium or cesium carbonate solution in de-ionized water, aiming at depositing an amount of alkali atoms that would roughly form one monoatomic layer over the tungsten surface (interior and exterior surface). Larger substrates are manufactured by commercial firms. Figure 1 shows a micro-photograph of a commercial $\mathrm{W}$ substrate used, after etching with a solution of $\mathrm{KOH}$ and $\mathrm{CuSO}_{4}$ to open the pores.

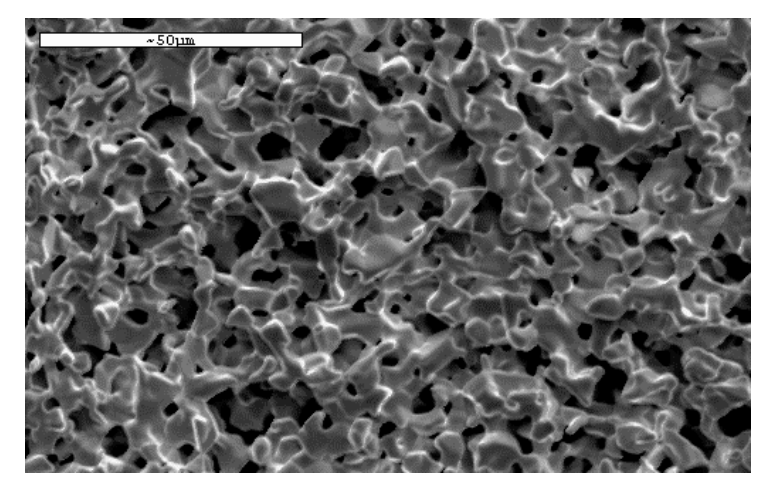

Figure 1: Micro-photograph of a $80 \%$ porous W substrate.

Upon the application of heat, the water evaporates and the carbonate breaks down releasing atomic K or Cs. In the case of potassium, the reactions are:

$$
\begin{aligned}
& \mathrm{K}_{2} \mathrm{CO}_{3} \rightarrow \mathrm{K}_{2} \mathrm{O}+\mathrm{CO}_{2} \\
& \mathrm{~K}_{2} \mathrm{O} \rightarrow 1 / 2 \mathrm{~K}_{2} \mathrm{O}_{2}+\mathrm{K} \\
& 2 \mathrm{~K}_{2} \mathrm{O}_{2} \rightarrow 2 \mathrm{~K}_{2} \mathrm{O}+\mathrm{O}_{2}
\end{aligned}
$$

Equations (1) and (3) proceed at temperatures above $600{ }^{\circ} \mathrm{C}$, while equation (2) at temperatures greater than $350{ }^{\circ} \mathrm{C}$. Once there is an adsorbed layer of the alkali metal on the tungsten surface, the interplay between the low ionization potential (I) of the adsorbed atom and the high work function $(\phi)$ of the substrate will favor a tunneling of the most loosely bound electron on the alkali into the metal, thus inducing a population of ions on the tungsten surface. The ratio of the ion to atom evaporation rates from the surface is given by the Saha-Langmuir Equation:

$$
\frac{v_{p}}{v_{a}}=\frac{1}{2} \exp \left[\frac{e(\phi-I)}{k T}\right]
$$

where "e" is the fundamental charge, $\mathrm{k}$ is Boltzmann's constant, $T$ is the substrate's temperature and $v_{p}$ and $v_{a}$ are the ion and neutral particle efflux rates per unit area 
respectively. The adsorbed ions on the surface can be extracted by the application of an electric field to produce a beam. Current output is further dependent on the surface coverage of the adsorbed layer. Since the alkali atoms can lower the substrate's work function, high current output require low alkali surface coverage (with an optimum value around $1 \%$ of a monolayer).

\subsection{Aluminosilicate Sources}

Alkali (K or Cs) aluminosilicates can emit alkali cations at high temperatures [2]. The ones used in our experiments are synthesized by mixing stoichiometric quantities of an alkali carbonate, silicon oxide and aluminum oxide. Significant care must be taken regarding the humidity control and thorough mixing. Equation (5) represents the aluminosilicate forming reaction. X represents either K or Cs.

$$
\begin{aligned}
& \mathrm{X}_{2} \mathrm{CO}_{3}+\mathrm{Al}_{2} \mathrm{O}_{3}+4 \mathrm{SiO}_{2} \underset{\Delta}{\longrightarrow} \\
& \mathrm{X}_{2} \mathrm{O} \cdot \mathrm{Al}_{2} \mathrm{O}_{3} \cdot 4 \mathrm{SiO}_{2}+\mathrm{CO}_{2} \uparrow
\end{aligned}
$$

The product is ground until it can be sifted through a 200-mesh wire sieve. A slurry, made of aluminosilicate powder and de-ionized water is poured onto the surface of the tungsten substrate. A lucite molding tool, matching the contour of the concave shaped substrate, is used to press the slurry and give it its final form with a desired thickness $(\sim 0.5 \mathrm{~mm})$. Slow drying is carried out in a chamber with humidity control. The final step is firing of the coated substrate at $1,580^{\circ} \mathrm{C}$ in a vacuum furnace. The temperature is controlled, both heating and cooling, at a rate of $\sim 10^{\circ} \mathrm{C} / \mathrm{min}$. Inadequate temperature control, or excessive temperature (above melting) may result in undesired loss of cations in the compound or surface distortion due to the molten compound's surface tension.
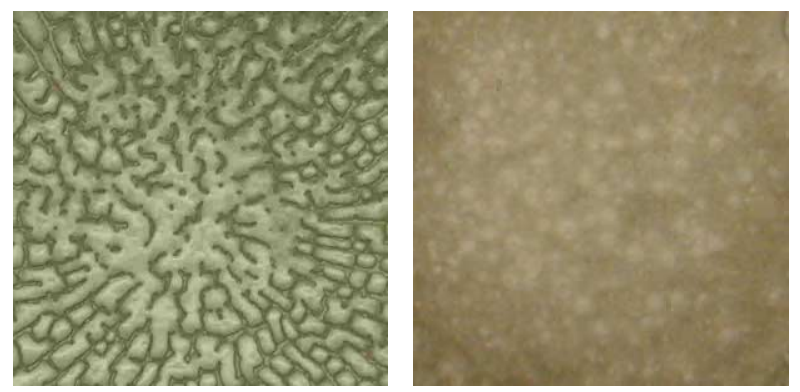

Figure 2. Surface defects due to inadequate drying process and too small particle size. Right: larger particle size and a slower drying rate.

Figure 2 shows the typical "micro-cracks" defects as a result of inadequate processing control (picture dimensions are $5 \mathrm{~cm} \times 5 \mathrm{~cm}$ ). The sample was coated to a smooth finish before firing using 400-mesh potassium aluminosilicate powder and quick dry. These defects are related to shrinkage problem during drying and surface tension after melting. Figure $2 b$, is an example of a good coating, done by using a coarser (200-mesh) powder and slow drying for less shrinkage.

\section{RESULTS \& DISCUSSION}

An ion gun capable of extracting high current densities $\left(\sim 100 \mathrm{~mA} / \mathrm{cm}^{2}\right)$ was built to test the performance of the small buttons. Two extraction voltage pulsers were used. A $70 \mathrm{kV}$ max, $2 \mu$ s pulse length and a $50 \mathrm{kV}$ max, $10 \mu \mathrm{s}$. The main parameter measured was the average J. Experimental results were accurately modeled by the code Trak 5.0 $0^{\mathrm{TM}}$, evidence that beams of the expected species were being produced. Figure 3 shows a collection of data for a $\mathrm{K}$ source. The expected behavior of the output, approaching the Child limit value can be observed. At lower temperatures, the source is emission limited, as it can not yield the output that the gun demands. J's of up to $89 \mathrm{~mA} / \mathrm{cm} 2$ of a space-charged limited $\mathrm{K}^{+}$beam were verified, as well as the capability to provide $10 \mu$ s flat pulses. Long pulse $(10 \mu \mathrm{s})$ duration was not tested at extraction voltages above $\sim 45 \mathrm{kV}$ (of $\mathrm{J} \cong 45 \mathrm{~mA} / \mathrm{cm}^{2}$ for a $\mathrm{K}$ beam). Lifetime tests measured the maximum average emission limited $\mathbf{J}$ as a function of time. Figure 4 shows an example of such lifetime tests for a $\mathrm{Cs}$ on $\mathrm{W}$ source. In the case presented, the Cs source was continuously operated and pulsed every $20 \mathrm{~s}$ with $2 \mu$ s pulses.

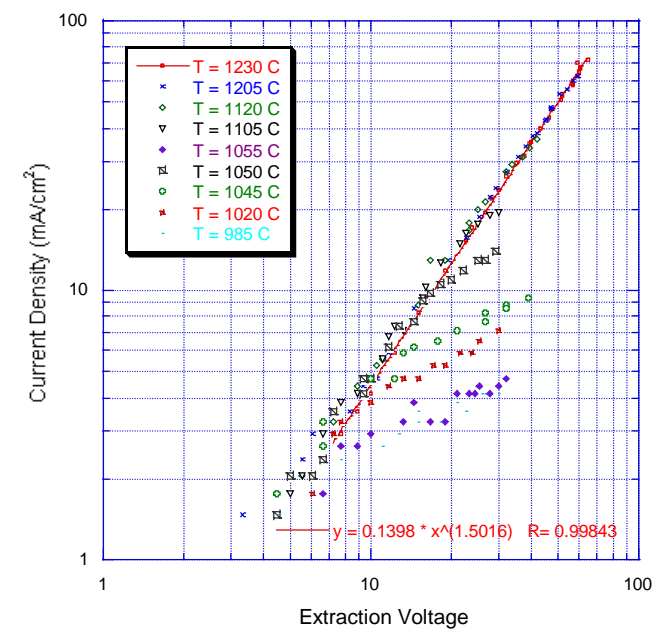

Figure 3: J-V curves at various temperatures for a $\mathrm{K}$ on $\mathrm{W}$

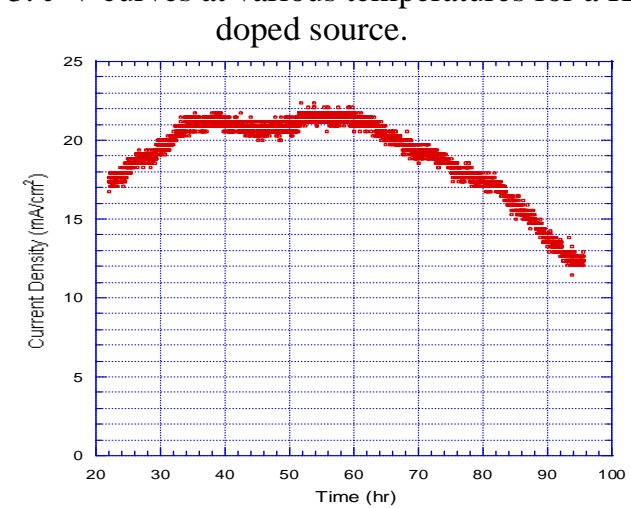

Figure 4: Life test for a Cs on W doped source. A fixed voltage of was kept, high enough to have emission limited output. Temperature kept constant at $1,100^{\circ} \mathrm{C}$. 


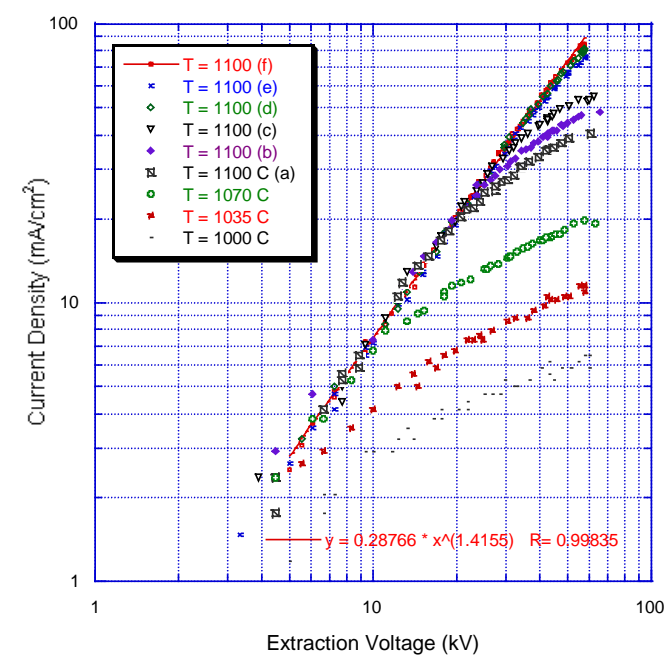

Figure 5: J-V curves at various temperatures for a $\mathrm{K}$ aluminosilicate source. Curves (b), (c), (d) and (e) were taken after 24, 48, 72 and 120 hours of operation at 1,100 ${ }^{\circ} \mathrm{C}$. Curve (f) was measured within an hour of curve (e).

Neutral K or Cs atoms are continuously being emitted from the hot surface. So far we do not have direct measurements of the neutral emission at the gun used in these experiments. Previous experiments [3] with a frontfeed Cs dispenser in a different ion gun yielded neutral evaporation rates of $1.7 * 10^{14}$ atoms $/\left(\mathrm{cm}^{2} * \mathrm{~s}\right.$ ) (or $27.2 \mu \mathrm{A} / \mathrm{cm}^{2}$ ). A constant neutral evaporation rate of this magnitude would deplete the total content of alkali atoms in the source within a period of $<1 \mathrm{hr}$. Further, if we consider the ion/neutral ratio given by Equation 4 at 1,100 ${ }^{\circ} \mathrm{C}$, and the measured ion output, the neutral efflux could be a factor of two larger. So there is an enormous disparity between the theoretical neutral emission and the neutral loss rates that estimated from the source's observed lifetime. This is observed also with $\mathrm{K}$ beams. This disparity could be explained by the utilization of an adjusted value for the substrate's work function. Contaminants in the vacuum system can affect the work function [4]; for instance, a pressure of $10^{-8}$ torr of oxygen may significantly increase the work function (up to $1.6 \mathrm{~V}$ with an adsorbed monolayer), thus decreasing the neutral efflux. An adsorbed quantity of oxygen on the substrate could be a product of the decomposition of the alkali carbonates used to dope the source, a phenomenon that was not observed with previous measurements on sources fed with alkali dispensers. Further research is required to confirm this phenomenon.

The aluminosilicate sources presented a similar behavior as the one observed with the doped sources in terms of the $\mathrm{J}-\mathrm{V}$ characteristics, but additionally, they presented a clear "activation" phenomenon. Figure 5 shows a typical aluminosilicate source data set. In this case, the curves labeled with letters are all at the same temperature but after different times of operation. Figure 6 shows the J-V characteristics of the same source after a

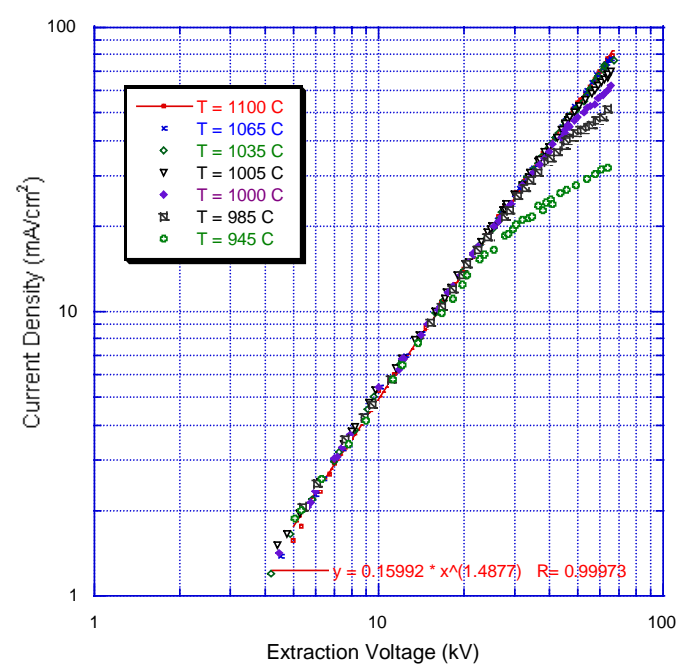

Figure 6: J-V curves at various temperatures for a fully activated $\mathrm{K}$ aluminosilicate source after 2 weeks of continuous operation.

continuous operation of 2 weeks. The increase in current output for a fixed temperature after activation can be easily observed. This activation period was observed to be about 5 days. The mechanism that allows for this activation is unknown. The lifetime of the aluminosilicate sources could not be properly assessed since they provided continuous operation at $\mathrm{T}=1,100{ }^{\circ} \mathrm{C}$ for more than 2.5 weeks without any decrease in output. The failure mode encountered was related to a detachment of the aluminosilicate layer from the tungsten substrate.

\section{CONCLUSIONS}

Given the high current density capability of the surface ionization sources, they can be used in HIF experiments. Doped source lifetime should be improved in future designs to provide sufficiently long lifetimes as observed lifetimes without re-doping are considered inadequate. This is despite the fact that much longer lifetimes than expected on a theoretical basis have been observed. Further experiments will be needed to assess the impact of oxygen or other contaminants on the source performance and lifetime. Aluminosilicate source final lifetime has not been assessed as further improvement in the fabrication technique is required.

\section{REFERENCES}

[1] Kwan, J.W. et al, "Design of a compact high current density injector for heavy ion fusion", these proceedings.

[2] Feeney, R.K.; Sayle W. E. and Hooper J. W., Rev. Sci. Instrum. 47, 964, (1976).

[3] Kwan, J.W.; Chupp W. W and Eylon S., Proc. Particle Accelerator Conf., Vancouver, May, (1997) p.2755

[4] Cho, A.Y.; Shelton, H. Cesium neutral and ion emission from carburized and oxygenated porous tungsten. AIAA J 2, 2135 (1964). 